\title{
Numerical simulation of the mixing process of the continuous mixer using the discrete element method
}

\author{
Tao Wang, a), Qiang $\mathrm{Xu}^{2}$, b) and Kaijie Zhang, ${ }^{2, c)}$ \\ ${ }^{1}$ School of Mechanical and Electonic Engineering, Nanjing Forestry University, Nanjing 210037, China. \\ ${ }^{2}$ School of Mechanical and Electonic Engineering, Nanjing Forestry University, Nanjing 210037, China. \\ a)1723438905@qq.com \\ b)714230833@qq.com \\ c)1018789454@qq.com
}

\begin{abstract}
This paper used the discrete element method to simulate the continuous mixing process and defined the time that the particles participated in mixing in the mixer was defined as the effective mixing time. The effects of different structures and rotational speeds on the mixing efficiency were studied by using the effective mixing time and dispersion coefficients, the function of the rotation speed and effective mixing time be fitted at the same time. In this research also found the appropriate outlet and rotation speed to make the mixer has a high mixing efficiency which was used in this paper. The research results can provide basis and reference for structural design of continuous mixer.
\end{abstract}

Key words: continuous mixer; DEM; rotation speed; effective mixing time;

\section{INTRODUCTION}

Mixing of powders and particles is a very important operation in industrial production which has been widely used in chemical, metallurgical, pharmaceutical, papermaking, food and wastewater treatment ${ }^{[1,2]}$. The structure and parameters of the mixing machinery directly affects whether the products which produced by the machinery meet the final production requirements, therefore, many scholars try to find out the relationship between mechanical structure and mixing parameter in various ways. Some scholars using different experimental approaches to analyze the mixing such as positron emission tomography(PET), positron emission particle tracking (PEPT) ${ }^{[3-6]}, \mathrm{X}$-ray tomography ${ }^{[7]}$ 和magnetic resonance imaging $(\mathrm{MRI})^{[8]}$. However, the conclusions of these experiments are only for the study of mixing mechanism, instead of associating the quality of the mixture and mechanical structure, the design of the mixing machinery depends on experience.

Computer numerical simulation technology helps researchers to overcome the problem of non - measurable caused by opaque materials with the great development of modern experimental techniques, computer modeling and computing ability, the study on the mixing machinery using numerical simulation become possible. At present, the numerical simulation on mixing of powders and particles usually based on discrete element method (DEM) which was first proposed by professor Cundall P.A ${ }^{[9]}$. The discrete element method has been supplemented and improved over the past few decades. Many scholars have carried on the numerical simulation to the mixer through the discrete element method. Maureen L. Rathod ${ }^{[10]}$ studied the effects of mixer speed, fluid inflow rate, and paddle angle on the mixing machinery, it was found that mixing efficiency increases with increase of mixing rate. Shahab Golshan ${ }^{[1]}$ et al. explored the sweeping rotation speed, primary rotation speed, impeller diameter on the mixing quality and mixing rate in nauta blenders, the maximum mixing rate was at $0.05 \mathrm{rad} / \mathrm{s}$. Meysam Alian and Simant R.Upreti ${ }^{[11]}$ researched on initial load, impeller speed, fill level, particle size, the conclusion was that the mixing time 
reduced with an increase in the rotational speed. Ruifang Wang ${ }^{[12]}$ simulated the randomness and mixing characteristics of soybean particles in horizontal rotating drum and analyzed the influence of rotor speed and load on particle random motion and mixing characteristics. It is found that increasing the rotating speed of the rotary drum is beneficial to enhance the mixing effect of the particles and accelerate the mixing rate. Guangyue Ren ${ }^{[13]}$ investigated the effects of mixing time, filling coefficient and blade speed on the Rotary Type Twin-shaft Paddle mixer and demonstrated that the influence of blade speed of the machine is the most significant. The above research is focused on the influence of the structure and parameters of batch mixer on the mixing mechanism, but research on continuous mixing machinery involving less.

This paper attempts to study the mixing progress of the continuous mixing machinery based on the practice on the results of previous studies and focuses on the impact of different speed and structure of mixed quality by using the discrete element method. At the same time, this paper puts forward the concept of effective mixing time for continuous mixing machine which can help the quantitative analysis between the parameters and the mixing quality on continuous mixed machinery, in addition this paper also optimizes the structure in order to provide more efficient for the future design of the continuous mixing mechanism.

\section{MODEL AND METHOD}

\section{DEM Introduction}

The DEM model was first proposed by Cundall P A ${ }^{[9]}$, which divide the research object into a separate unit, using dynamic relaxation method and static relaxation method of iterative method iterative calculation according to Newton's laws of motion and interactions between cells. determine the stress and displacement of each the time step of all units. Particles are mainly influenced by its own gravity and the normal and tangential contact force between particles in the process of movement.

According to Newton's second law, linear motion equations for each particle ${ }^{[14]}$

$$
m_{i} \frac{d v_{i}}{d t}=m_{i} g+\sum_{j=1}^{n_{i}}\left(F_{n, i j}+F_{t, i j}\right)
$$

The particle is also subjected to tangential and frictional torque, which is the rotational equation

$$
I_{i} \frac{d \omega_{i}}{d t}=\sum_{j=1}^{n_{i}}\left(T_{t, i j}+T_{r, i j}\right)
$$

$T_{t, i j}$ is tangential moment of particle $i$, the, $\operatorname{Tr}, i j$ is the rolling friction moment of particle $i$.

The simulation is based on the mixing simulation software EDEM and ignores the simulation of adhesion and liquid bridge force between particles due to the simulation particle are dry particles. Based on the above analysis, the Hertz-Mindlin (no slip) contact mechanics model is chose ${ }^{[15]}$.

\section{Geometry and Simulation Conditions}

The mixer consists of a cylindrical stationary body (1) which is equipped with an inlet on the top and an outlet on the right, 12 mixer impellers(2) and a mixer(stirring) shaft(3), as shown in figure 1 . The particles enter the mixer from the inlet and starts to mixing through the mixer impellers, after a period of time, the material outflow from the outlet. 

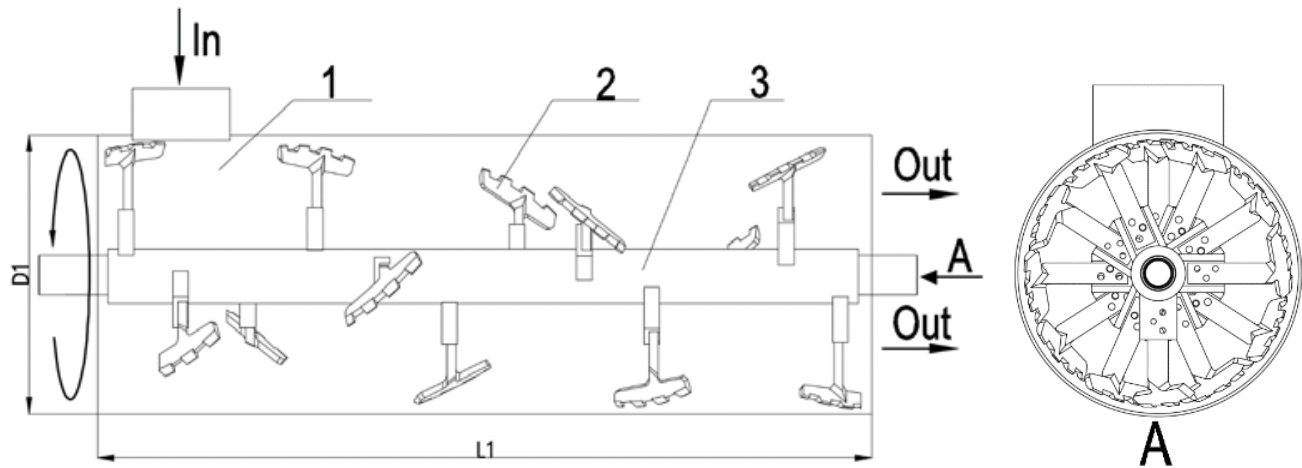

FIGURE 1 the Structure of Mixer

The DEM simulation parameters (gathered from ${ }^{[15]}$ ) are listed in Table 1 and the simulation conditions are summarized as flowed:

The way that particles flows into the mixer: Continuous inflow

The way that particles flows out of the mixer: Continuous outflow

TABLE 1. Parameter used in the simulation

\begin{tabular}{cc}
\hline Parameter & Value \\
\hline Particle Poisson's radio & 0.25 \\
Particle shear modulus & $4.5 \mathrm{e}+10 \mathrm{~Pa}$ \\
Particle density & $5200 \mathrm{~kg} / \mathrm{m}^{3}$ \\
Vessel Poisson's radio & 0.3 \\
Vessel shear modulus & $7 \mathrm{e}+10 \mathrm{~Pa}$ \\
Vessel density & $7800 \mathrm{~kg} / \mathrm{m}^{3}$ \\
Particle-Particle restitution coefficient & 0.2 \\
Particle-Vessel restitution coefficient & 0.2 \\
Particle-Particle static friction coefficient & 0.545 \\
Particle-Vessel static friction coefficient & 0.6 \\
Particle-Particle rolling friction coefficient & 0.01 \\
Particle-Vessel rolling friction coefficient & 0.01 \\
\hline
\end{tabular}

\section{Mixing Assessment}

The mixing uniformity is one of the main technical indicators to measure the mixing effect and the material mixing uniformity can be inferred by calculating the dispersion coefficient CV (\%) of one kind of particles in the mixture. The smaller the dispersion coefficient, the higher the uniformity of mixing ${ }^{[17]}$.

The coefficient of variation is calculated as follows.

$$
\bar{X}=\frac{X_{1}+X_{2}+X_{3}+\cdots X_{N}}{N}
$$

Standard deviation S:

$$
S=\sqrt{\frac{\left(X_{1}-X_{2}\right)^{2}+\left(X_{2}-X\right)^{2}+\left(X_{3}-X\right)^{2}+\cdots+\left(X_{N}-X\right)^{2}}{N-1}}
$$

dispersion coefficient CV (\%)

$$
C V=\frac{S}{\bar{X}} \times 100
$$

The continuous mixer was mainly explored in this paper which was different from the batch mixer. In mixing process of the continuous mixer, the particles continuously inflow from the inlet and outflow from the outlet, the time that the particles participated in mixing was a fixed value if the speed, filling rate, particle properties and other parameters constant. In this paper, the time that the particles participated in mixing in the mixer was defined as the effective mixing time, so that the reader does not mix with the mixing time described in other literature. 
Due to the different mixing parameters corresponding to different effective mixing time, it was possible to have the following 3 conditions without changing the structure of the mixer and other parameters

1) When the mixture flows out from the outlet, it can meet the requirements just right. It is the ideal condition with the highest mixing efficiency.

2) The mixture has reached the requirements of the mixing uniformity, but still stay in the mixer instead of flowing out from the outlet, namely excessive mixing of particles. In this situation, the mixture meet the mixing requirement, but wasting resources and reducing the mixing efficiency.

3) The mixture is not mixed evenly from the outlet. In this situation, the mixture can't meet the requirement of the mixing uniformity.

In order to ensure the optimal mixing efficiency, it is necessary to study the effective mixing time of continuous mixer. In this paper, the kind of the outlet and rotation speed will be studied to explore the relationship between these factors and the efficiency of the continuous mixer. effective mixing time and effect of different speeds on the mixing time.

\section{NUMERICAL SIMULATION OF MIXING PROGRESS}

\section{Particles Mixing Progress}

This paper used discrete element method to simulate the particle mixing process, in order to facilitate the study of effective mixing time and mixing uniformity at the same time, respectively, particle A (6mm radius) and particle B (radius $5 \mathrm{~mm}$ ) were fed at the entrance, the ratio of particle A and particle B is 1:2. In this paper, particle B can be used as a missing agent to study the effective mixing time in this mixer and also can be used to research on the mixing uniformity of this mixer, therefore, particle $\mathrm{A}$ and particle $\mathrm{B}$ have the same parameters except the diameters of particle A and particle B. A portion of the particle A will be filled in the mixer in advance. Monitoring the outflow of the outlet particles, when the particle B flows out from the outlet in a steady amount, this time is the effective mixing time of this mixer.

The mixing process of the particle A and particle B as shown in Figure 2. Particle A and particle B were continuously adding into the entrance, at the time of $1 \mathrm{~s}$, particle B accumulated in the entrance below, at the time of $5 \mathrm{~S}$, particle B were slowly moved to the exit and started mixing with particle A at the same time. At the time of 10s, a portion of the particles B has moved to the tail of the mixer. Due to the rotation of the mixer impellers, the particle $\mathrm{B}$ simultaneously moves along the axial direction and the radial direction of the mixer. The concentration of particulate $\mathrm{B}$ in the mixer exhibits a process of aggregation and dispersion from the inlet to the outlet, which indicates that the particle B is mixed with the particle A and after a certain period of time, the particle A and particle B flow out from the outlet of the mixer

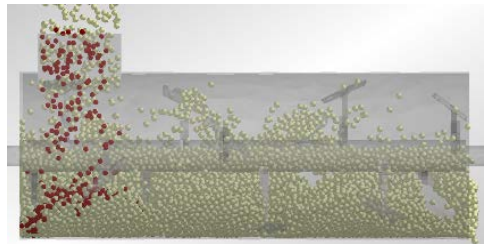

$1 \mathrm{~s}$

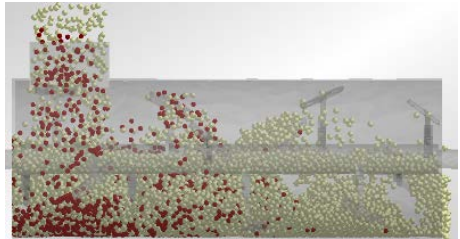

$5 s$

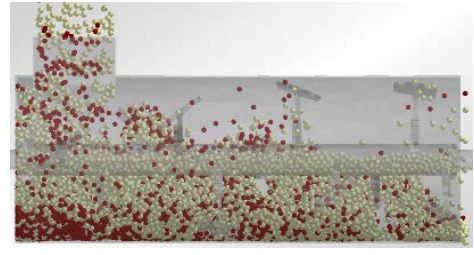

$10 \mathrm{~s}$

FIGURE 2. the process of the mixing

The effective mixing time can be seen in Figure 3, where the abscissa represents time, and the ordinate represents the number of particles in the $\mathrm{B}$ corresponding to the number of seconds. As can be seen in the 13s, the particle B outflow tends to be stable, indicating that in this case, the effective mixing time of the mixer is $13 \mathrm{~s}$. 


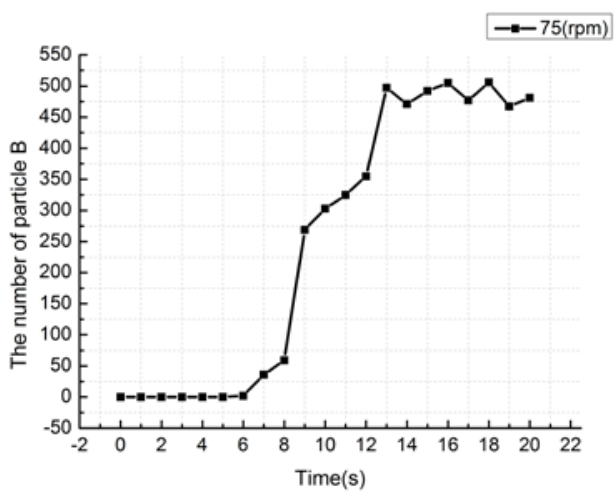

FIGURE 3. the number of the particle B flow out from outlet per second with time

\section{The Influence of the Outlet}

Through the research on the particle field of the mixer, it can be found that the particles in the mixer are constantly rising and falling due to the rotation of the mixer impellers and the gravity of the particles, finally, these particles were outflow from export. The export was divided into 16 regions respectively numbered as shown in Fig.5, the particles were mainly outflow from the right(region 3,4,7,8,11,12) and the bottom(region 14,15,16). Adding baffle in outlet can block a portion of the particles, making the retention time of the particles in the mixer longer than no baffle, so that the effective mixing time of material growth

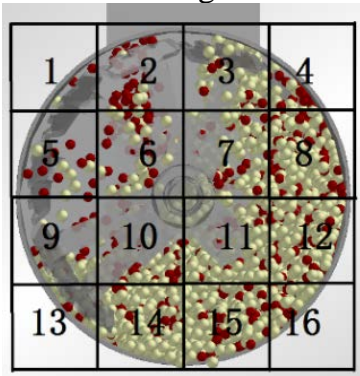

FIGURE.4 the outlet was divided into 16 regions

\section{The Influence of the Rotation Speed}

The particle velocity along the axial and radial will be increased along with the increases of the rotation speed of the mixer impellers. The bigger the velocity of particles in the mixer is, the stronger the particle movement is, the more favorable to the mixing of particles. As the particle velocity increases along the direction of the mixing shaft, the effective mixing time became shorter which were not conducive to the mix of particles. While on the other hand, the effective mixing time longer, the material in the mixer mixing degree may be worse. Therefore, it is necessary to study the rotating speed of the mixer

\section{RESULT AND DISCUSSION}

\section{The Relationship between outlet and the Effective Mixing Time}

According to the outflow of particles from the outlet, selecting region 12,14,15,18 as the outlet, the outlet as shown in Figure 6b. Selecting region 12,15,18 as the outlet, the outlet shown in Figure 6c. 


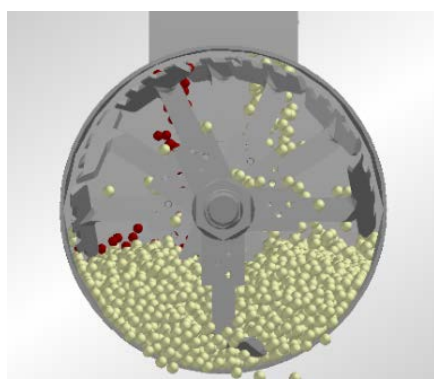

a

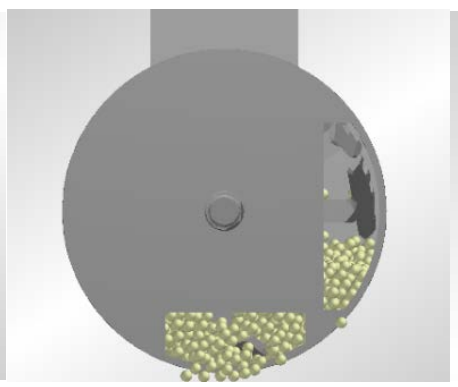

$\mathrm{b}$

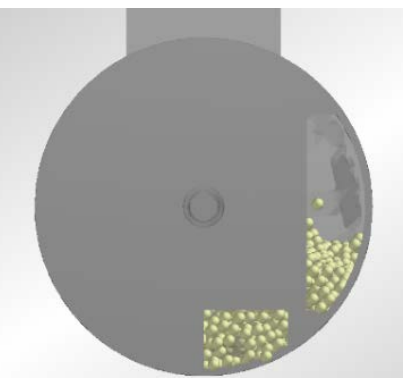

C

FIGURE.5 3 kind of outlet

Through numerical simulation, it can be seen that different outlet corresponds to different effective mixing time when the rotational speed is 75rpm. The effective mixing time of different kind of outlet can be seen in Figure 6. The effective mixing time of outlet a, b and c are 13s, 22s and 30s. The effective mixing time became longer from the outlet a to outlet b to outlet c because of the different blocking region which was used to obstruct a portion of particles outflow from the outlet.

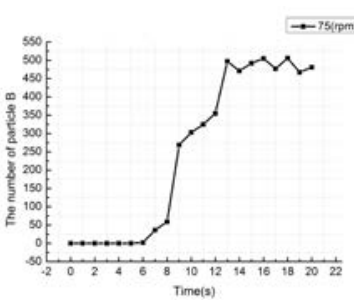

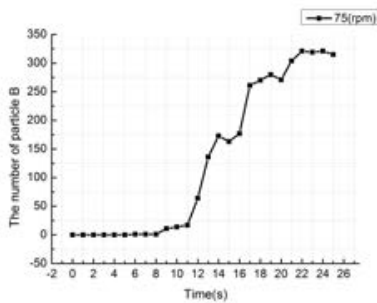

$\mathrm{b}$

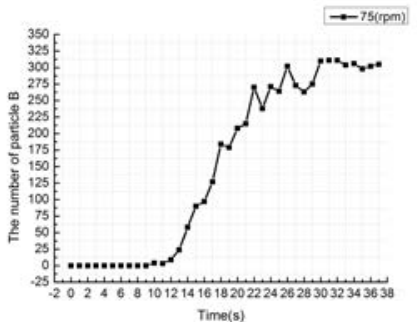

c

FIGURE.6 the number of the particle B flow out from outlet per second with time at different outlet

The final purpose of the mixer is to mix evenly in the shortest time, so the study of mixing uniformity is also necessary. Through the simulation of different structure of the mixer, the dispersion coefficient can be calculated, the data as shown in Table 2. By comparing the data obtained from the simulation at the same rotation speed, it can be found that the mixing efficiency of mixer with outlet a and outlet b isn't high because the mixture outflow from the outlet dose not mix evenly. The mixer with outlet c maybe mix evenly or does not mix evenly, however, if increasing the blocking region, the number of particles outflow must be reduced causing the reduction of production efficiency. In order to further study the mixing time and the effective mixing time, this paper considered that the outlet $\mathrm{c}$ is a better one and no longer study the effective mixing time by changing the outlet.

Table 2 Different dispersion coefficient with different outlet

\begin{tabular}{cccc}
\hline Outlet & a & b & c \\
\hline Dispersion coefficient CV (\%) & 0.47 & 0.33 & 0.21 \\
\hline
\end{tabular}

\section{The Relationship between Rotation Speed and the Effective Mixing Time}

In this section, relationship between the rotation speed of mixer and effective mixing time will be mainly studied. The bigger the velocity of particles in the mixer is, the stronger the particle movement is, the more favorable to the mixing of particles. In order to research the relationship between the rotation speed and effective mixing time, 5 cases which has the different rotation speed (60rpm, 75rpm, 90rpm,105rpm, 120rpm) with the same structure were simulated 

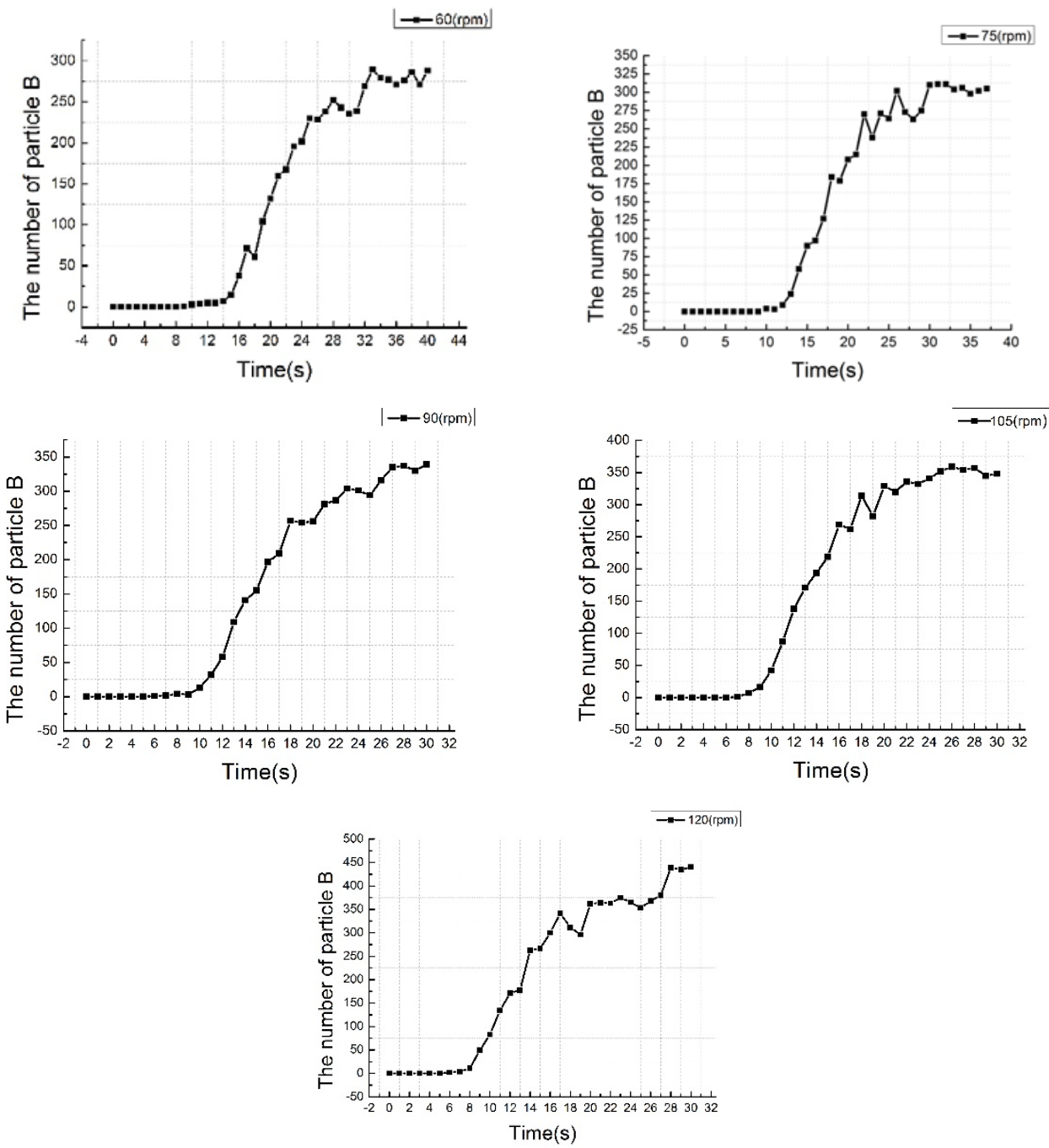

FIGURE 7 the number of the particle B flow out from outlet per second with time at different rotation speed

According to the results of the simulation, the effective mixing time with different rotating speed were shown in figure 7 . The value of the dispersion coefficient and effective mixing time were listed in the table

TABLE 3 the value of the dispersion and effective mixing time with different rotation speed

\begin{tabular}{cccccc}
\hline Rotation speed(rpm) & $\mathbf{6 0}$ & $\mathbf{7 5}$ & $\mathbf{9 0}$ & $\mathbf{1 0 5}$ & $\mathbf{1 2 0}$ \\
\hline Dispersion coefficient CV (\%) & 0.206 & 0.262 & 0.208 & 0.112 & 0.129 \\
Effective mixing time(s) & 32 & 30 & 27 & 24 & 28 \\
\hline
\end{tabular}

From the numerical simulation, it can be found that when the rotation speed was 60rpm, the effective mixing time was the longest and when the rotation speed was 105rpm, the effective mixing time was the shortest. With the increasing of rotation speed, effective mixing time of the mixer reduced, then increased at the time of 120rpm. It is found that the movement of particles were more severe and the diffusion degree of particles is higher in the mixer with the rotation speed of 120rpm comparing with the mixer with the rotation speed of 75rpm. Because of the outlet of the mixer, the particles were blocked more (as shown in figure 8) by comparing the mixer with 75rpm and 120rpm which increased the effective mixing time. 


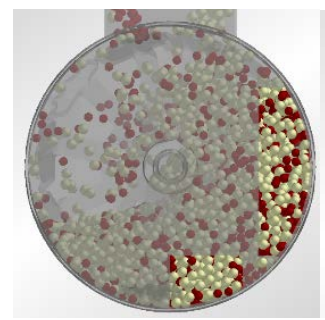

$75 \mathrm{rpm}$

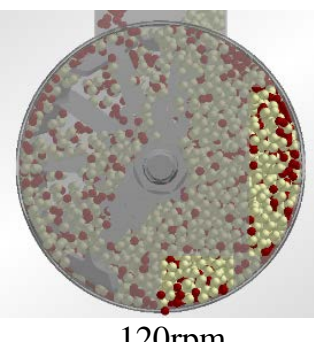

120rpm

FIGURE 8 the outlet at the rotation speed of 75rpm and 120rpm

The dispersion coefficient decreased gradually and tend to be stable when the rotation speed between 70rpm and 120rpm, which shows that the mixing uniformity increased as the rotation speed increased. When the rotation speed was 105rpm, the value of dispersion coefficient was the minimum, it means that when the rotational speed was 105rpm, the mixing uniformity was the highest. Considering the effective mixing time and the dispersion coefficient, the efficiency of the mixer was the best with the outlet c and the rotation speed of 105rpm.

Fitting the function of effective mixing time and the rotation speed according to the simulation as shown in figure9

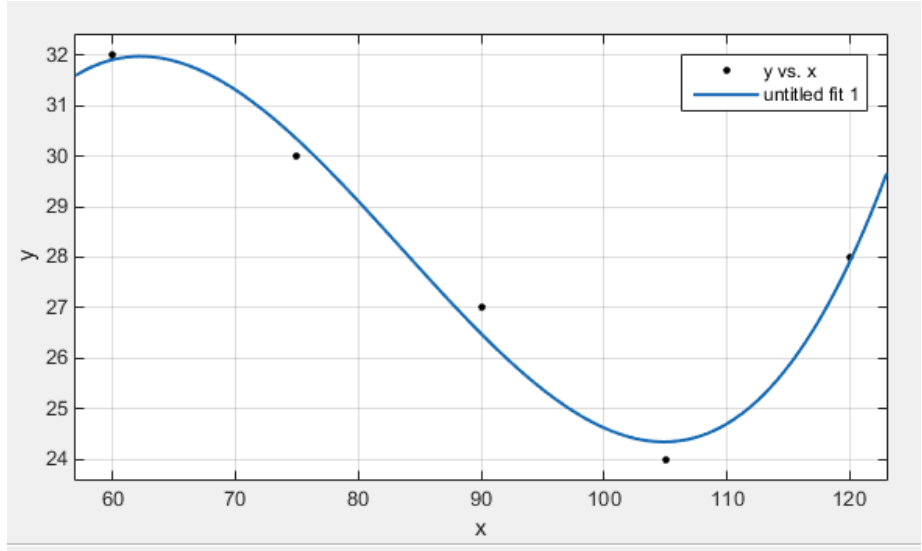

FIGURE 9. Fitting curve of effective mixing time and rotational speed,

$\mathrm{X}$ axis represents rotation speed (rpm) and Yaxis represents effective mixing time(s)

Getting the function:

$$
f(t)=0.0002 r^{3}-0.0495 r^{2}+3.87 r-64.66
$$

$f(t)$ is the effective mixing time(s), $r$ is the rotation speed (rpm).

\section{CONCLUSION}

This paper simulated the progress of the continuous mixer using the discrete element method. Since the feed and discharge of the continuous mixer is continuous, the particles can only stay in the mixer for a certain period of time which defined the effective mixing time in this paper. Effective mixing time was considered as the inherent parameter of continuous mixer together with the dispersion coefficient to explore the mixing efficiency of the continuous mixer. This paper investigated the effect of outlet and rotation speed on the continuous mixer and optimized the structure of the continuous mixer, meanwhile, fitted the function of the rotation speed and the effective mixing time. The following conclusions can be drawn:

a) The particles were mainly outflow from the right (region 3,4,7,8,11,12) and the bottom (region 14,15,16) as shown in figure 4.

b) The effective mixing time became longer and the mixing uniformity became higher from the outlet a to outlet b to outlet $\mathrm{c}$ at the same rotation speed.

c) The effective mixing time decreased with the increase of the rotation speed when the rotation speed between 60rpm and 105rpm, the effective mixing time increased with the increase of the rotation speed when the rotation speed between 105rpm and 120rpm. 
d) The dispersion coefficient decreased gradually and tend to be stable when the rotation speed between 70rpm and 120rpm at the same structure of the continuous mixer.

e) The efficiency of the mixer was the best with the outlet c and the rotation speed of 105rpm.

f) The function of the effective mixing time and the rotation speed was:

$$
f(t)=0.0002 r^{3}-0.0495 r^{2}+3.87 r-64.66
$$

$f(t)$ is the effective mixing time(s), $r$ is the rotation speed (rpm).

\section{ACKNOWLEDGMENTS}

Corresponding author: Qiang Xu (associate professor)

\section{REFERENCES}

1. Golshan S, Zarghami R, Norouzi H R, et al, Granular mixing in nauta blenders, J. Powder Technology.2017,305:279-288.

2. Basinskas G, Sakai M, Numerical study of the mixing efficiency of a ribbon mixer using the discrete element method, J. Powder Technology. 2016,287:380-394.

3. Lim S Y, Davidson J F, Forster R N, et al, Avalanching of granular material in a horizontal slowly rotating cylinder: PEPT studies, J. Powder Technology. 2003,138(1):25-30.

4. Laurent B F C, Cleary P W, Comparative study by PEPT and DEM for flow and mixing in a ploughshare mixer, J. Powder Technology. 2012,228:171-186.

5. Zhu H P, Zhou Z Y, Yang R Y, et al, Discrete particle simulation of particulate systems: A review of major applications and findings, J. Chemical Engineering Science. 2008,63(23):5728-5770.

6. Portillo P M, Vanarase A U, Ingram A, et al, Investigation of the effect of impeller rotation rate, powder flow rate, and cohesion on powder flow behavior in a continuous blender using PEPT, J. Chemical Engineering Science,2010,65(21):5658-5668.

7. Liu R, Yin X, Li H, et al, Visualization and quantitative profiling of mixing and segregation of granules using synchrotron radiation X-ray microtomography and three dimensional reconstruction., J. International Journal of Pharmaceutics. 2013,445(1-2):125-133.

8. Sommier N, Porion P, Evesque P, et al, Magnetic resonance imaging investigation of the mixing-segregation process in a pharmaceutical blender, J. International Journal of Pharmaceutics. 2001,222(2):243.

9. Cundall P A, Strack O D L, A discrete numerical model for granular assemblies, J. Géotechnique. 1979,30(3):331-336.

10. Rathod M L, Kokini J L, Effect of mixer geometry and operating conditions on mixing efficiency of a nonNewtonian fluid in a twin screw mixer, J. Journal of Food Engineering. 2013,118(3):256-265.

11. Alian M, Ein-Mozaffari F, Upreti S R, Analysis of the mixing of solid particles in a plowshare mixer via discrete element method (DEM), J. Powder Technology. 2015,274:77-87

12. Wang Ruifang, Li Zhanyong et al, Simulation on Random Motion and Mixing Characteristic for Soybean in Rotary Drum, J. Transactions of The Chinese Society of Agricultural Machinery. 2013(06):93-99.

13. Ren Guang-yue, YU Qing-long et al, Experimental study on mixing performance of the twin-shaft paddle rotating mixer, J. Journal of Agricultural Mechanization Research. 2004(06):167-170.

14. Gao Hongli, Jiang Maoqiang et al, Study on simulation of parameters optimization of ball mills with DEM, J. MINING \& PROCESSING EQUIPMENT. 2010(11):74-77.

15. Liu Yang Han, Yan-Long et al, Numerical simulation on stirring motion and mixing characteristics of ellipsoid particles, J. Acta Physica Sinica. 2015(11):258-265.

16. Zhou Zhiyong, Blending Process Simulation and Structure Optimization of Strong Mixer Based on DEM, D. Xiangtan University. 2014.

17. He Feng, Wu Lei et al, Rapid detection method of pellet raw material mixing uniformity, J. China Metallurgy. 2016(02):39-43. 